\title{
S \\ Nonlinear single Compton scattering of an electron wave packet
}

\author{
A. Angioi, F. Mackenroth, ${ }^{*}$ and A. Di Piazza ${ }^{\dagger}$ \\ Max-Planck-Institut für Kernphysik, Saupfercheckweg 1, 69117 Heidelberg, Germany
}

(Received 8 February 2016; published 2 May 2016)

\begin{abstract}
Nonlinear single Compton scattering has been thoroughly investigated in the literature under the assumption that the electron initially has a definite momentum. Here, we study a more general initial state and consider the electron as a wave packet. In particular, we investigate the energy spectrum of the emitted radiation and show that, in typical experimental situations, some features of the spectra shown in previous works are almost completely washed out. Moreover, we show that, at comparable relative uncertainties, the one in the momentum of the incoming electron has a larger impact on the photon spectra at a fixed observation direction than the one on the laser frequency.
\end{abstract}

DOI: 10.1103/PhysRevA.93.052102

\section{INTRODUCTION}

According to classical electrodynamics, a charged particle (an electron, for definiteness) accelerated by a background electromagnetic field emits radiation [1]. In the underlying quantum theory, QED, the radiation process is rather described as the emission of photons by the electron [2,3]. Due to energy-momentum conservation, a free electron is stable and cannot emit photons. The scattering of an electron with a single photon is known as (linear) Compton scattering. In general, the simultaneous interaction of an electron with many photons is suppressed by the appearance in the interaction probabilities of a corresponding power of the fine-structure constant $\alpha_{\mathrm{QED}} \approx 1 / 137 \ll 1$. However, if the electron interacts with a coherent collection of photons, such as those in a laser beam, the effective coupling strength appearing in perturbative expansions is not just $\alpha_{Q E D}$ but also depends on the typical amplitude and angular frequency of the laser field [4]. Qualitatively, it is clear that a laser field characterized by an amplitude $\mathcal{E}$ and by an angular frequency $\omega$ is able to transfer to the electron (charge $e<0$ and mass $m$ ) a number of photons of the order of

$$
\xi=\frac{|e| \mathcal{E}}{\omega m c}
$$

in the typical QED length $\lambda_{C}=\hbar / m c \approx 3.9 \times 10^{-11} \mathrm{~cm}$ (Compton wavelength) [4-6]. Thus, at $\xi \gtrsim 1$, the probability for the electron of exchanging more than one photon with the laser field is not suppressed and the laser-electron interaction has to be taken into account exactly in the calculations. From a classical point of view, the condition $\xi \gtrsim 1$ corresponds to the onset of relativistic effects in the electron dynamics, which render the latter nonlinear with respect to the laser amplitude. Now, if the electron enters a plane-wave field with a four-momentum $p^{\mu}=(\varepsilon / c, \boldsymbol{p})$, with $\varepsilon=\left(m^{2} c^{4}+|\boldsymbol{p}|^{2} c^{2}\right)^{1 / 2}$, and in the process of photon emission it absorbs $\xi$ laser photons, due to the Doppler effect, the typical energy $\hbar \omega^{\prime}$ of the emitted photon is of the order of $\chi \varepsilon$, where

$$
\chi=\frac{(p k)}{m \omega} \frac{\mathcal{E}}{\mathcal{E}_{c r}},
$$

\footnotetext{
*Present address: Department of Applied Physics, Chalmers University of Technology, Gothenburg, Sweden.

†dipiazza@mpi-hd.mpg.de
}

with $k^{\mu}=(\omega / c, \boldsymbol{k})$ being the plane wave's four-wave-vector and $\mathcal{E}_{c r}=m^{2} c^{3} / \hbar|e| \approx 1.3 \times 10^{16} \mathrm{~V} / \mathrm{cm}$ being the so-called "critical field" of QED [4,5]. The above estimate of the typical energy of the emitted photon is valid for $\chi \lesssim 1$. A constant and uniform electric field of the order of $\mathcal{E}_{c r}$ provides an $e^{-}-e^{+}$ pair with an energy comparable to its rest energy $2 m c^{2}$ on a distance of the order of $\lambda_{C}$, such that the QED vacuum becomes unstable in the presence of such a strong field under $e^{-}-e^{+}$pair creation [7-9]. The parameter $\chi$ controls the importance of photon recoil, which becomes essential at $\chi \gtrsim 1$. The emission of a single photon in the regime $\xi, \chi \gtrsim 1$ is known as nonlinear single Compton scattering and it has been studied thoroughly in the literature [10-25].

Although initial electron wave packets have been considered in some studies about Thomson scattering [26], and the general problem of the radiation emitted by a classical distribution of charges is a well-known problem in the free-electron-laser community [27], in the study of nonlinear Compton scattering, to the best of our knowledge, the initial state of the electron has been mostly taken as having a definite momentum. In experiments, however, an electron in a beam has some characteristic indeterminacy in momentum and is localized to some extent; motivated by this fact, we consider below that the electron is initially in a superposition of states of different momenta, i.e., in a wave packet, and, among other aspects, we study whether it is possible to observe interference effects among different components of the wave packet. We do this in the framework of strong-field QED within the Furry picture [4-6,28]. In Ref. [29] a scalar QED calculation with an initial particle described by a wave packet shows how, for nonlinear single Compton scattering in a monochromatic plane-wave electromagnetic field, the different components of the electron wave packet do not interfere. This result has been extended in Ref. [30] to spinor QED in pulsed fields. We show in the following a different derivation of the same result and, in addition, we investigate in detail the effects of the initial electron's wave packet on the emitted radiation.

Peak laser intensities have been recently increasing dramatically due to the development of two techniques: chirped pulse amplification (CPA) [31] and optical parametric chirped pulse amplification (OPCPA) [32]. All of today's most intense lasers, such as Vulcan [33], Astra-Gemini [34], HERCULES [35], the Berkeley Lab Laser Accelerator (BELLA) [36], and planned ones, such as the Extreme Light Infrastructure 
[37], the High-Power Laser Energy Research facility [38], APOLLON [39], and the Exawatt Center for Extreme Light Studies (XCELS) [40], are based on one or the other of these techniques. Both CPA and OPCPA generate, after the amplification of an initial pulse, an ultrashort laser pulse; it is thus likely that these kind of pulses will be adopted in experiments to probe the nonlinear QED regime. Thus, we consider the laser field in our calculations to be an ultrashort pulse.

To date, the record for the highest laser intensity ever achieved is held by the HERCULES facility, which reached a peak intensity of $2 \times 10^{22} \mathrm{~W} / \mathrm{cm}^{2}(\xi \approx 70$ at $\hbar \omega=1.55 \mathrm{eV})$, and lasers with peak intensity $\xi \gtrsim 1$ are readily available in many facilities. It is harder, however, to reach values of the parameter $\chi$ close to unity. Starting from Eq. (2), and substituting the previously given definition of $\mathcal{E}_{c r}$, one can write $\chi=\xi \hbar(p k) / m^{2} c^{2}$; the factor $\hbar(p k) / m^{2} c^{2}$ makes it necessary, in order to have a $\chi$ close to unity at optical frequencies, to use ultrarelativistic electrons (even for large values of $\xi \sim 100$ ). Nowadays, ultrarelativistic electron beams can be conveniently produced also at laser facilities via the wakefield acceleration technique [41,42].

Although intense pulses are usually focused almost down to the diffraction limit, we model them as plane waves. This approximation is valid if the electron collides nearly head-on with the laser field and almost at the focus of the latter, provided that the transverse excursion of the electron is much smaller than the laser waist size, which occurs if $\xi m c^{2} \ll \varepsilon[3,43]$. Within the plane-wave approximation, the approach based on the Furry picture can be conveniently applied because the Dirac equation in a plane-wave field can be solved exactly. Approximate solutions can be also found, however, for a field of more complex structure, such as a Gaussian laser beam, if the conditions $\xi \gg 1$ and $\xi m c^{2} \ll \varepsilon$ are fulfilled [44].

In most of the numerical work performed to obtain the results in this paper, one of the main challenges is to perform integrals of highly oscillating functions; typical quadrature schemes cannot be adopted, since they become more and more inaccurate as the frequency of the oscillations of the integrand increases. Thus, we use Filon's method $[45,46]$ to deal with this type of integral. The basic idea behind it is to put a highly oscillating integral in the form $\int_{\mathscr{J}} d x f(x) e^{i a x}$, where $f(x)$ is a smooth and sufficiently-well-behaved function, $a \gg 1$ is a constant, and $\mathcal{I}$ is an interval in $\mathbb{R}$; then divide $\mathcal{I}$ in some subintervals $\left\{\mathcal{I}_{n}, n \in \mathbb{N}\right\}$ sufficiently small that, in each of them, the function $f(x)$ can be accurately approximated with a quadratic polynomial. Then, in each subinterval the starting integrals are approximated by a weighted sum of terms each having the form $\int_{\mathscr{I}_{n}} d x x^{j} e^{i a x}$, where $j \in\{0,1,2\}$, and each of these integrals can be evaluated analytically. The advantage of this method is that the accuracy of the estimate increases with increasing $a$.

This paper is organized as follows: In Sec. II, we present the general theory of the scattering of an electron in a superposition of states with different momenta and a short intense laser pulse, and we show that interference effects among states with different momenta are not present. In Sec. III, we study the particular case of an electron wave packet colliding head-on with a laser pulse and of normally distributed longitudinal momentum, while in Sec. IV we investigate the more general

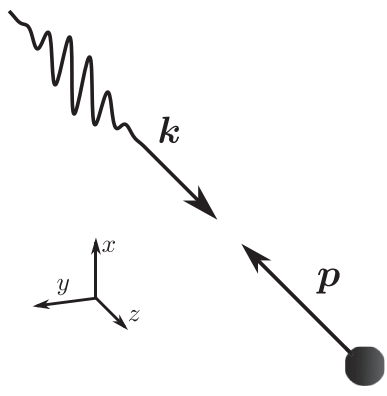

FIG. 1. Representation of the frame of reference employed.

case where there is also an indeterminacy on the transverse components of the momentum. Through the rest of the article, natural units ( $\hbar=c=1)$ are adopted, and the electromagnetic units used are such that the QED coupling constant is $\alpha_{Q E D}=$ $e^{2}(\approx 1 / 137)$.

\section{THEORY}

In the computation of nonlinear single Compton scattering rates, perturbative approaches with respect to the laser field can quickly become impractical when a sufficiently strong incoming electromagnetic field is considered. In fact, as we mentioned in the introduction, for an incoming laser field such that $\xi \gtrsim 1$, the exchange of many photons between the laser and the electron becomes important and perturbative calculations up to a very high order would be necessary. Typically, however, such intense fields consist of an enormous number of coherent photons; this makes it possible [3] to neglect the quantum nature of the background field and to treat it as a classical given electromagnetic field. By working within this approximation, one can split the electromagnetic field four-vector potential into two parts: a classical part, that accounts for the intense laser field, and a quantized part, that accounts for all the other excitations of the electromagnetic field, i.e., the radiation emitted by the electron. After that, the electron-positron field is quantized by taking into account exactly the background laser field. This is the so-called Furry picture of QED [3,28], which we mentioned in the introduction, and all the following calculations are performed within this formalism.

We assume that the incoming laser field is described by the linearly polarized plane-wave four-vector potential

$$
A^{\mu}(\eta)=\mathcal{A}^{\mu} \psi(\eta)
$$

Here, $\mathcal{A}^{\mu}=(0, \mathcal{A})$ is a constant four-vector, where $\mathcal{A}$ defines the laser polarization, with amplitude $\mathcal{A}=\mathcal{E} / \omega$ related to the peak laser intensity $I$ as $I=\omega^{2} \mathcal{A}^{2} / 4 \pi=\mathcal{E}^{2} / 4 \pi$, and $\psi(\eta)$ is a function of the laser phase $\eta=(k x)$ describing the shape of the plane wave and such that $|\psi(\eta)| \sim|d \psi(\eta) / d \eta| \leqslant 1$. It is convenient to use a frame of reference in which one of the spatial axes (in our case the $z$ axis, for the sake of definiteness) is directed along $\boldsymbol{k}$, and another one (without loss of generality, we can choose $x$ ) is directed along the same direction as $\mathcal{A}$ (see Fig. 1). Thereby, we have $\eta=\omega(t-z)=\omega \varphi$, where $\varphi=$ $t-z$. It is also useful to introduce a coordinate $T=(t+z) / 2$, linearly independent of $\varphi, x$, and $y$, and the quantities $\varphi, T$, $x$, and $y$ provide the so-called light-cone coordinates of the 


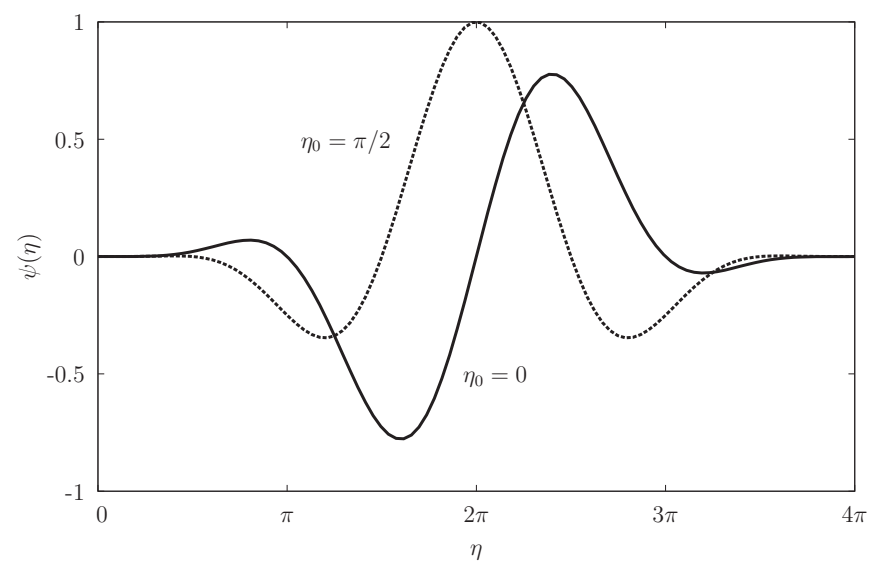

FIG. 2. The function $\psi(\eta)$ for a two-cycle laser pulse $\left(n_{C}=2\right)$ and two choices of the carrier-envelope phase $\eta_{0}$. The solid curve corresponds to $\eta_{0}=0$, while the dotted curve corresponds to $\eta_{0}=$ $\pi / 2$.

space-time point $x^{\mu}$ (the factor $1 / 2$ in the definition of $T$ is arbitrary and we have chosen it in order for the Jacobian of the transformation from Cartesian coordinates to light-cone coordinates to be unity). In the following, we define the (minus) contravariant component of any four-vector $q^{\mu}$ to be $q^{-}=q^{0}-q^{3}$.

In the expression of $A^{\mu}(\eta)$ we introduce the shape function $\psi(\eta)$ in order to model short laser pulses; a typically chosen [18] shape function $\psi(\eta)$ for this purpose is (see Fig. 2)

$$
\psi(\eta)= \begin{cases}\sin ^{4}\left(\frac{\eta}{2 n_{C}}\right) \sin \left(\eta+\eta_{0}\right) & \text { if } \eta \in\left[0,2 \pi n_{C}\right] \\ 0 & \text { otherwise. }\end{cases}
$$

In this parametrization of the laser field we introduced the parameters $n_{C}$, the number of cycles contained in the laser pulse, and $\eta_{0}$, the carrier-envelope phase (CEP) of the laser pulse.

In all the numerical calculations in the following, we will chose $\eta_{0}=0, n_{c}=2$, and $\omega=1.55 \mathrm{eV}$.

In the Furry picture, the states of the electron are described by the solutions of the Dirac equation in the presence of the background field; if this field is the plane wave $A^{\mu}(\eta)$, these solutions (known as Volkov states) are given by $[3,47]$

$$
\begin{aligned}
\Psi_{p, \sigma}(x)= & {\left[1+\frac{e}{2(k p)} \not k A(\eta)\right] u_{p, \sigma} } \\
& \times e^{-i p x-i \int_{-\infty}^{\eta}\left[\frac{e}{(k p)}\left(p A\left(\eta^{\prime}\right)\right)-\frac{e^{2}}{2(k p)} A^{2}\left(\eta^{\prime}\right)\right] d \eta^{\prime}},
\end{aligned}
$$

where the slash on a four-vectorial quantity is a shorthand notation for a contraction of that four-vector with the Dirac matrices $\gamma^{\mu}$, that is, $\not l=\gamma^{\mu} a_{\mu}$ and $u_{p, \sigma}$ is a positive-energy spinor solution of the free Dirac equation $(\not p-m) u_{p, \sigma}=$ 0 , with $\bar{u}_{p, \sigma} u_{p, \sigma}=2 m\left(\bar{u}_{p, \sigma}=u_{p, \sigma}^{\dagger} \gamma^{0}\right)$. The Volkov state $\Psi_{p, \sigma}(x)$ is characterized by the four-momentum $p^{\mu}=(\varepsilon, \boldsymbol{p})$ $\left[\varepsilon=\left(m^{2}+|\boldsymbol{p}|^{2}\right)^{1 / 2}\right]$ and by the spin quantum number $\sigma$ at $t \rightarrow-\infty$ (these are the so-called Volkov in-states, although Volkov out-states only differ from the in-states by a phase independent of the coordinates). The Volkov states in Eq. (5) are normalized as

$$
\int d^{3} x \Psi_{p^{\prime}, \sigma^{\prime}}^{\dagger}(x) \Psi_{p, \sigma}(x)=(2 \pi)^{3}(2 \varepsilon) \delta\left(\boldsymbol{p}-\boldsymbol{p}^{\prime}\right) \delta_{\sigma, \sigma^{\prime}} .
$$

As we have mentioned in the introduction, we consider an electron state which is a wave packet made of a superposition of Volkov states with different momenta and a given spin number $\sigma$ :

$$
\Phi_{\sigma}(x)=\int \frac{d^{3} p}{(2 \pi)^{3}(2 \varepsilon)} \rho(\boldsymbol{p}) \Psi_{p, \sigma}(x) .
$$

Here, $\rho(\boldsymbol{p})$ is a complex-valued, scalar weighting function; in order for the state $\Phi_{\sigma}(x)$ to be normalized to unity, $\rho(\boldsymbol{p})$ needs to be normalized in a covariant way as

$$
\int \frac{d^{3} p}{(2 \pi)^{3}(2 \varepsilon)}|\rho(\boldsymbol{p})|^{2}=1 .
$$

The leading-order $S$-matrix element relative to the process of the emission of a photon, with wave four-vector $k^{\prime \mu}=$ $\left(\omega^{\prime}, \boldsymbol{k}^{\prime}\right)$ and polarization four-vector $\epsilon_{l}^{\prime \mu}$, by an electron in the initial state $\Phi_{\sigma}(x)$ is

$$
\begin{aligned}
S_{f i}= & -i e \sqrt{4 \pi} \int d^{4} x \frac{d^{3} p}{(2 \pi)^{3}(2 \varepsilon)} \\
& \times \rho(\boldsymbol{p}) \bar{\Psi}_{p^{\prime}, \sigma^{\prime}}(x) \notin_{l}^{\prime *} e^{i k^{\prime} x} \Psi_{p, \sigma}(x) .
\end{aligned}
$$

We notice that, among the space-time coordinates, the integrand in Eq. (9) depends nontrivially only on $\varphi$, while on the other three space-time coordinates we have integrals that evaluate to three delta functions. It is thus possible [18] to write $S_{f i}$ in the form

$$
\begin{aligned}
S_{f i}= & -i e \sqrt{4 \pi}(2 \pi)^{3} \int \frac{d^{3} p}{(2 \pi)^{3}(2 \varepsilon)} \\
& \times \rho(\boldsymbol{p})\left(\bar{u}_{p^{\prime}, \sigma^{\prime}} M_{f i} u_{p, \sigma}\right) \delta^{(-, x, y)}\left(p-k^{\prime}-p^{\prime}\right) ;
\end{aligned}
$$

here, $\delta^{(-, x, y)}\left(p-k^{\prime}-p^{\prime}\right)$ is a three-dimensional Dirac delta that ensures the conservation of the three contravariant components,$- x$, and $y$ of the total four-momentum and

$$
\begin{gathered}
M_{f i}=\notin_{l}^{\prime *} f_{0}+e\left(\frac{\mathcal{A} k \notin_{l}^{* *}}{2\left(k p^{\prime}\right)}+\frac{\notin_{l}^{* *} k \mathcal{A}}{2(k p)}\right) f_{1}-\frac{e^{2} \mathcal{A}^{2}\left(k \epsilon_{l}^{\prime *}\right) \not k}{2(k p)\left(k p^{\prime}\right)} f_{2}, \\
f_{j}=\int_{-\infty}^{+\infty} d \eta \psi^{j}(\eta) e^{i \int_{-\infty}^{\eta} d \eta^{\prime}\left[\alpha \psi\left(\eta^{\prime}\right)+\beta \psi^{2}\left(\eta^{\prime}\right)+\gamma\right]}
\end{gathered}
$$

In Eq. (12) we introduced the three parameters [48]

$$
\begin{gathered}
\alpha=e\left[\frac{\left(p^{\prime} \mathcal{A}\right)}{\left(k p^{\prime}\right)}-\frac{(p \mathcal{A})}{(k p)}\right], \\
\beta=-\frac{e^{2} \mathcal{A}^{2}}{2} \frac{\left(k^{\prime} k\right)}{(k p)\left(k p^{\prime}\right)}, \\
\gamma=\frac{\left(p k^{\prime}\right)}{\left(p^{\prime} k\right)} .
\end{gathered}
$$


In order to compute emission rates, it is necessary to calculate the square modulus of $S_{f i}$ :

$$
\begin{aligned}
\left|S_{f i}\right|^{2}= & 4 \pi e^{2} \int \frac{d^{3} p}{(2 \varepsilon)} \frac{d^{3} \tilde{p}}{(2 \tilde{\varepsilon})} \rho^{*}(\tilde{\boldsymbol{p}}) \rho(\boldsymbol{p})\left(\bar{u}_{p^{\prime}, \sigma^{\prime}} M_{f i} u_{\tilde{p}, \sigma}\right)^{*} \\
& \times\left(\bar{u}_{p^{\prime}, \sigma^{\prime}} M_{f i} u_{p, \sigma}\right) \\
& \times \delta^{(-, x, y)}\left(p-k^{\prime}-p^{\prime}\right) \delta^{(-, x, y)}\left(\tilde{p}-k^{\prime}-p^{\prime}\right) .
\end{aligned}
$$

The integrations in Eq. (16) are along the components of $\boldsymbol{p}$ and $\tilde{\boldsymbol{p}}$ in Cartesian coordinates, while one of the delta functions in Eq. (16) is expressed in terms of light-cone coordinates. An easy way to perform these integrations is to change the measure for each momentum integration from $d p_{x} d p_{y} d p_{z}=$ $d^{2} p_{\perp} d p_{z}$ to $d^{2} p_{\perp} d p^{-}$; the Jacobian one has to insert for this transformation is $\varepsilon / p^{-}$. Thus one can start from Eq. (16), change the integration measure to $d^{2} p_{\perp} d p^{-} d^{2} \tilde{p}_{\perp} d \tilde{p}^{-}$, perform the integrations in $\tilde{p}$ (which are just integrations of delta functions), and change back the measure to $d^{3} p$; this gives

$$
\begin{aligned}
\left|S_{f i}\right|^{2}= & 4 \pi e^{2} \int \frac{d^{3} p}{(2 \varepsilon)\left(2 p^{-}\right)}|\rho(\boldsymbol{p})|^{2}\left|\bar{u}_{p^{\prime}, \sigma^{\prime}} M_{f i} u_{p, \sigma}\right|^{2} \\
& \times \delta^{(-, x, y)}\left(p-k^{\prime}-p^{\prime}\right) .
\end{aligned}
$$

The unpolarized emission rate is obtained by integrating $\left|S_{f i}\right|^{2}$ over the electron's final momentum and on the wave vector of the emitted photon, and by summing over the final electron spin and photon polarization, and averaging over the initial electron spin [2,3]:

$$
\begin{aligned}
d W= & \frac{d^{3} k}{(2 \pi)^{3}\left(2 \omega^{\prime}\right)} \int \frac{d^{3} p^{\prime}}{(2 \pi)^{3}\left(2 \varepsilon^{\prime}\right)} \frac{d^{3} p}{(2 \varepsilon)\left(2 p^{-}\right)} \\
& \times 4 \pi e^{2}|\rho(\boldsymbol{p})|^{2} \delta^{(-, x, y)}\left(p-k^{\prime}-p^{\prime}\right) \\
& \times \frac{1}{2} \sum_{\sigma, \sigma^{\prime}, l}\left|\bar{u}_{p^{\prime}, \sigma^{\prime}} M_{f i} u_{p, \sigma}\right|^{2} .
\end{aligned}
$$

The integral on $d^{3} p^{\prime}$ can be readily evaluated with the same change of integration measure previously mentioned. By writing $d^{3} k^{\prime}=\omega^{\prime 2} d \omega^{\prime} d \Omega^{\prime}$ and remembering that the emission rate and the energy emission rate are related by $d E=\omega^{\prime} d W$, it is possible to write the angular differential emission rate as

$$
\begin{aligned}
\frac{d E}{d \omega^{\prime} d \Omega^{\prime}}= & \int \frac{d^{3} p}{(2 \pi)^{3}(2 \varepsilon)}|\rho(\boldsymbol{p})|^{2} \frac{e^{2} \omega^{\prime 2}}{2(4 \pi)^{2} p^{-} q^{-}} \\
& \times \sum_{\sigma, \sigma^{\prime}, l}\left|\bar{u}_{q, \sigma^{\prime}} M_{f i} u_{p, \sigma}\right|^{2}
\end{aligned}
$$

where $q^{\mu}$ is a four-vector such that $q^{-}=p^{-}-k^{\prime,-}, q_{x, y}=$ $p_{x, y}-k_{x, y}^{\prime}$, and $q^{+}=\left(q^{0}+q^{3}\right) / 2=\left(m^{2}+q_{x}^{2}+q_{y}^{2}\right) / 2 q^{-}$ $\left(q^{2}=m^{2}\right)$. Equation (19) can be easily identified as the incoherent average over the modulus squared of $\rho(\boldsymbol{p})$ of the well-known expression of the differential angular energy emission rate for a nonlinear single Compton scattering event of an electron with definite initial four-momentum $p^{\mu}$ and final four-momentum $q^{\mu}[14,18,48]$ :

$$
\frac{d E_{p}}{d \omega^{\prime} d \Omega^{\prime}}=\frac{e^{2} \omega^{\prime 2}}{2(4 \pi)^{2} p^{-} q^{-}} \sum_{\sigma, \sigma^{\prime}, l}\left|\bar{u}_{q, \sigma^{\prime}} M_{f i} u_{p, \sigma}\right|^{2} .
$$

Thus, there are no quantum interference effects between initial states of the electron having different values of momentum.
The physical reason behind the absence of interference is that, in principle, by measuring the final state of the electron and of the emitted photon one can retrieve the initial momentum of the electron, and so the initial state of the electron among the ones contained in the initial superposition.

The results we presented so far allow us to state that, as far as one is interested in nonlinear single Compton scattering rates, the state of the initial electron can be described equivalently either with a superposition of states like the one in Eq. (7) or as a statistical mixture

$$
\hat{\rho}_{\sigma}=\int \frac{d^{3} p}{(2 \pi)^{3}(2 \varepsilon)}|\rho(\boldsymbol{p})|^{2}\left|\Psi_{p, \sigma}\right\rangle\left\langle\Psi_{p, \sigma}\right|,
$$

where the weighting function $\rho(\boldsymbol{p})$ is the same as Eq. (7) and $\Psi_{p, \sigma}(x)=\left\langle x \mid \Psi_{p, \sigma}\right\rangle$.

\section{ELECTRON WAVE PACKETS WITH NORMALLY DISTRIBUTED LONGITUDINAL MOMENTUM}

After describing the theory for arbitrary superpositions of Volkov states (for a given spin quantum number), in this section and in the next we make an explicit choice of $\rho(\boldsymbol{p})$. Let the initial state of the electron be a superposition of states with momenta always directed almost in the opposite direction of the laser wave vector $\boldsymbol{k}$ (for the choice of the frame of reference we adopted in Sec. II, i.e., the momenta $\boldsymbol{p}$ are all directed almost along the negative $z$ direction). In particular, we assume that the distribution of the momenta is a triple Gaussian distribution, with average momentum $\overline{\boldsymbol{p}}=\left(0,0, \bar{p}_{z}\right)$, with $\bar{p}_{z}<$ 0 , and with variance $\sigma_{p_{T}}^{2}$ along the $x$ and $y$ direction and $\sigma_{p_{z}}^{2}$ along the $z$ direction; thus the initial wave packet is given by

$$
\begin{aligned}
\Phi_{\sigma}(x)= & \int \frac{d^{3} p}{(2 \pi)^{3}(2 \varepsilon)} \frac{1}{\sigma_{p_{T}} \sqrt[4]{\sigma_{p_{z}}^{2}(2 \pi)^{3}}} \\
& \times e^{-\frac{\left(p_{z}-\bar{p}_{z}\right)^{2}}{4 \sigma_{p_{z}}^{2}}} e^{-\frac{p_{x}^{2}+p_{y}^{2}}{4 \sigma_{p_{T}}^{2}}} \Psi_{p, \sigma}(x) .
\end{aligned}
$$

In the present section the transverse variance $\sigma_{p_{T}}^{2}$ is assumed to be sufficiently small, so that all transverse momenta $\left(p_{x}, p_{y}\right)$ in Eq. (19) can be set equal to zero [except that in the exponential in Eq. (22)]. Thus, the electron effectively collides head-on with the laser beam.

In order to understand the modifications brought about by the electron being described by the wave packet in Eq. (22), we plot in Fig. 3 the emission spectrum in the forward (negative $z$ ) direction for an incoming electron with definite momentum with components $p_{x}=p_{y}=0$, and $p_{z}=-4.2 \mathrm{GeV}(\varepsilon \approx 4.2 \mathrm{GeV})$ [42] interacting with a laser of intensity $I \approx 4.3 \times 10^{20} \mathrm{~W} / \mathrm{cm}^{2}$. The above parameters correspond to $\xi=10$ and $\chi \approx 0.50$.

The spectra in the regime of $\left|p_{z}\right| \gg m$ and $\xi \gg 1$ exhibit a large number of narrow peaks. The position of the peaks depends on the momentum of the electron; in particular, from Fig. 4 one can deduce that, as the electron's initial momentum increases in modulus, these peaks will be shifted toward higher frequencies.

These shifts depend on the position of the peaks itself, i.e., different peaks are shifted by a different amount, when changing $p_{z}$. More specifically, by changing $p_{z}$ by the same amount, the higher peak frequencies will be shifted more than the lower ones. The above results can be easily explained as a result of the 


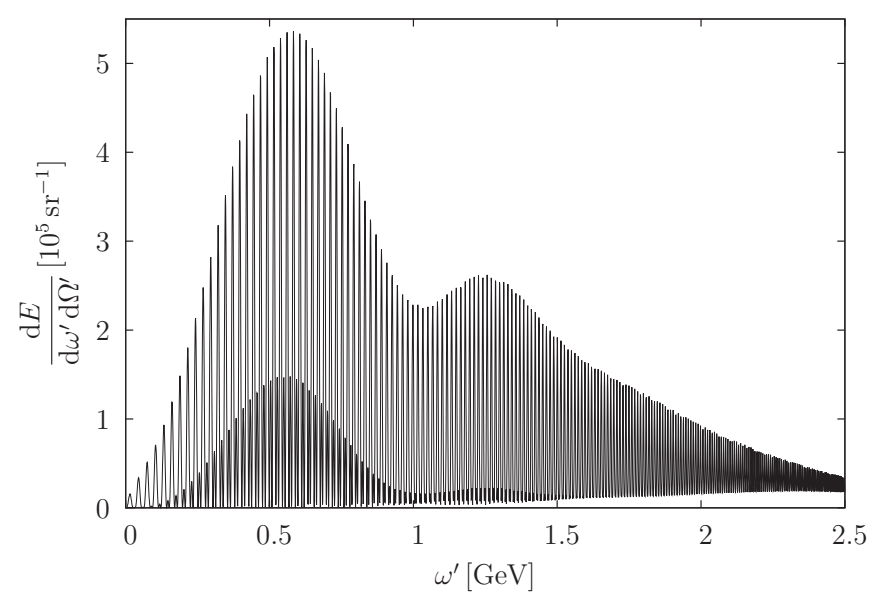

FIG. 3. Energy emission spectrum along the negative $z$ direction for an incoming electron with definite initial momentum $\boldsymbol{p}=(0,0,-$ $4.2 \mathrm{GeV}$ ) interacting with a laser of intensity $I \approx 4.3 \times 10^{20} \mathrm{~W} / \mathrm{cm}^{2}$.

Doppler effect. For the sake of simplicity we consider here the idealized case of a monochromatic laser field (with laser photon energy $\omega$ ). In this case, in fact, the frequency of the $n$th harmonic emission along the negative $z$ direction is given by [4]

$$
\begin{aligned}
\omega_{n}^{\prime} & =\frac{n(p k)}{\left(p n^{\prime}\right)+\left(n+\frac{m^{2} \xi^{2}}{4(p k)}\right)\left(k n^{\prime}\right)} \\
& =\frac{n \omega\left(\varepsilon-p_{z}\right)^{2}}{m^{2}\left(1+\frac{\xi^{2}}{2}\right)+2 n \omega\left(\varepsilon-p_{z}\right)} \\
& =\frac{\zeta_{n}}{1+2 \zeta_{n}}\left(\varepsilon-p_{z}\right),
\end{aligned}
$$

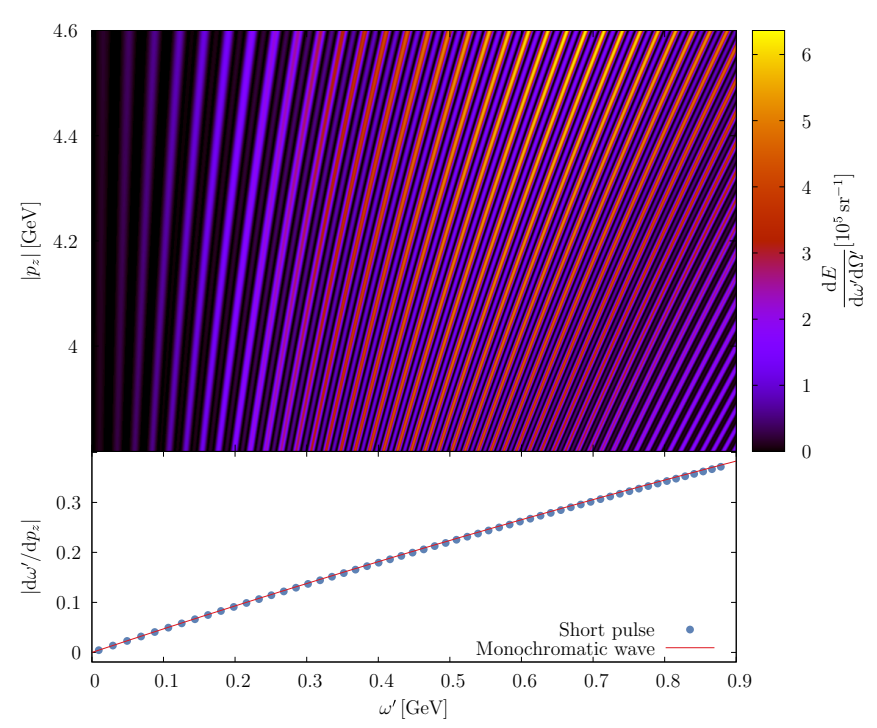

FIG. 4. Change of emission spectrum for an electron with definite initial momentum $\left(0,0, p_{z}\right)$ as a function of $\left|p_{z}\right|$ (Fig. 3 corresponds to a section of the upper part of this figure for $p_{z}=-4.2 \mathrm{GeV}$ ). In the range considered, the position of the peaks increases linearly with $p_{z}$, albeit with different slopes depending on the position of the peak. Some of these slopes were computed numerically and are shown in the bottom part of the plot (blue dots), together with the same quantity computed analytically for a monochromatic pulse (red continuous line). where $n^{\prime \mu}=\left(1, \boldsymbol{k}^{\prime} / \omega^{\prime}\right)=(1,0,0,-1)$ and where we have introduced the dimensionless parameter

$$
\zeta_{n}=\frac{n \omega\left(\varepsilon-p_{z}\right)}{m^{2}\left(1+\frac{\xi^{2}}{2}\right)} .
$$

By means of a first-order expansion with respect to the shift $\Delta p_{z}$, we can estimate the relative shift of these frequencies when slightly changing the value of $p_{z}$ :

$$
\frac{\Delta \omega_{n}^{\prime}}{\omega_{n}^{\prime}}=\frac{1}{\omega_{n}^{\prime}} \frac{\partial \omega_{n}^{\prime}}{\partial p_{z}} \Delta p_{z}=-2 \frac{1+\zeta_{n}}{1+2 \zeta_{n}} \frac{\Delta p_{z}}{\varepsilon} .
$$

In the case of an ultrarelativistic electron and in the relevant regime $\xi \gg 1$, it is $\varepsilon \approx\left|p_{z}\right|$ and $\zeta_{n} \approx 2 n \chi / \xi^{3}$, such that we obtain

$$
\frac{\Delta \omega_{n}^{\prime}}{\Delta\left|p_{z}\right|} \approx 4 \frac{\zeta_{n}\left(1+\zeta_{n}\right)}{\left(1+2 \zeta_{n}\right)^{2}} .
$$

As can be easily shown, the quantity $\Delta \omega_{n}^{\prime} / \Delta\left|p_{z}\right|$ increases monotonically with the harmonic number, in agreement with the findings in Fig. 4.

Notice that Eq. (26) is valid only for a monochromatic laser field, whereas we are interested here in the case of short pulses, i.e., pulses also characterized by a certain spread $\Delta \omega$ around a central angular frequency $\omega$. It is thus interesting to compare the relative shift due to an uncertainty of $p_{z}$ to the one due to an indeterminacy on the value of $\omega$. In analogy to what we have discussed for Eq. (25), one can derive a similar relation for a variation $\Delta \omega$ of the laser angular frequency. By adding the resulting expression to Eq. (25) and by assuming again that $\left|\bar{p}_{z}\right| \gg m$ and $\xi \gg 1$, it is possible to obtain the first-order relative variation of $\omega_{n}^{\prime}$ with respect to the relative variations of $\omega$ and $p_{z}$ as

$$
\frac{\Delta \omega_{n}^{\prime}}{\omega_{n}^{\prime}} \approx \frac{1}{1+2 \zeta_{n}} \frac{\Delta \omega}{\omega}+2 \frac{1+\zeta_{n}}{1+2 \zeta_{n}} \frac{\Delta\left|p_{z}\right|}{\left|p_{z}\right|} .
$$

Since $\zeta_{n}>0$ it is clear that, for comparable relative variations in $\omega$ and $p_{z}$, the induced shift due to the spread in the incoming electron momentum is larger.

From the aforementioned properties of the emitted photon's spectrum of a monochromatic initial electron we can infer the final spectrum when the state $\Phi_{\sigma}(x)$ of Eq. (7) is considered, since the emission spectrum resulting from that state, as it was shown above, is a weighted average of monochromatic emission spectra with different $p_{z}$. The sharp peaks present in the spectrum for a fixed value of $p_{z}$ will be differently shifted and will tend to fill the valleys present in the spectrum relative to another value of $p_{z}$; when averaging many of these spectra, the net effect is a smoothing of the final spectra and a decrease of the yield as compared with the latter obtained at the peaks in the monochromatic case.

Moreover, we have already mentioned the fact that the shift induced by the spread in the electron momentum is larger for higher emission frequencies. Thus, the portion of the spectrum that will be smoothed earlier, i.e., even for relatively small values of $\sigma_{p_{z}}$, is that at high frequencies of the emitted photon. Indeed, this is the result we obtain in Fig. 5, where the final photon energy spectrum for different values of $\sigma_{p_{z}}$ is plotted [the numerical parameters are the same as in Fig. 3 and the average value of the initial momentum of the electron is 


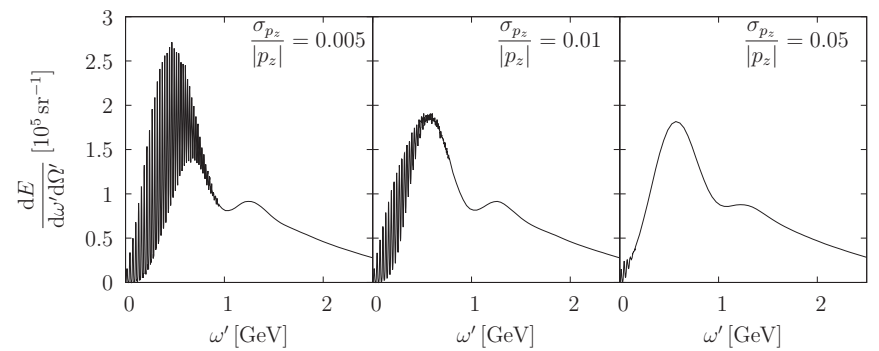

FIG. 5. Emission spectra along the negative $z$ direction for an electron wave packet with $\bar{p}=(0,0,-4.2 \mathrm{GeV})$ interacting with a laser pulse of peak intensity $I \approx 4.3 \times 10^{20} \mathrm{~W} / \mathrm{cm}^{2}$ for different values of the spread of the longitudinal momentum.

$\overline{\boldsymbol{p}}=(0,0,-4.2 \mathrm{GeV})]$. We have chosen values of the standard deviation $\sigma_{p}$ equal to $0.5 \%, 1 \%$, or $5 \%$ of the incoming momentum, corresponding to $21 \mathrm{MeV}, 42 \mathrm{MeV}$, or $210 \mathrm{MeV}$, respectively. Even when the relative indeterminacy on the momentum is only $0.5 \%$, we can see that the height of the highest peaks is reduced by a factor of about two, and all the oscillatory features at $\omega^{\prime} \gtrsim 1 \mathrm{GeV}$ are completely washed out (see Figs. 3 and 5). For larger values of $\sigma_{p_{z}}$, these effects are even more evident also for the lowest part of the spectrum. Concerning the choice of $\sigma_{p_{z}}$ and, in general, of the properties of the wave packet $\Phi_{\sigma}(x)$, a comment is in order. In fact, in general, the state $\Phi_{\sigma}(x)$ describes a single electron. The properties of the corresponding wave packet depend on how the electron is produced and accelerated [49] and are in principle different from, for example, the corresponding properties of an electron bunch. However, in our case, as we have seen, the spectra for the state $\Phi_{\sigma}(x)$ coincide with those obtained by considering a corresponding electron bunch with an average electron number equal to unity. In this respect, the values of the momentum spreads are chosen according to the features of electron beams, which can be obtained presently experimentally [42].

\section{MULTIVARIATE GAUSSIAN WAVE PACKETS}

We now turn our attention to the experimentally more realistic situation of an electron wave packet that can also have nonzero components of the transverse momentum. Our choice for the initial state is as in Eq. (22) but this time the variance $\sigma_{p_{T}}^{2}$ is assumed not to be small. Also in this case, as we did in the previous section, we first consider how the spectrum of electrons initially in a Volkov state in a monochromatic field is modified as a function of the components of the initial momentum. Then, starting from those considerations, we focus on the case of an electron wave packet in a short laser pulse.

In order to understand how the emission spectrum is altered by the possibly nonzero value of the transverse components of the initial momentum, we show how the harmonic frequencies along the negative $z$ direction are shifted as the transverse momentum $p_{T}=\left(p_{x}^{2}+p_{y}^{2}\right)^{1 / 2}$ varies. We can thus proceed in analogy to the derivation of Eq. (26). The starting point is the initial form of $\omega_{n}^{\prime}$ in Eq. (23), which can be rewritten in the more convenient form

$$
\omega_{n}^{\prime}=\frac{n \omega\left(\varepsilon-p_{z}\right)^{2}}{m^{2}\left(1+\frac{\xi^{2}}{2}\right)+p_{T}^{2}+2 n \omega\left(\varepsilon-p_{z}\right)},
$$

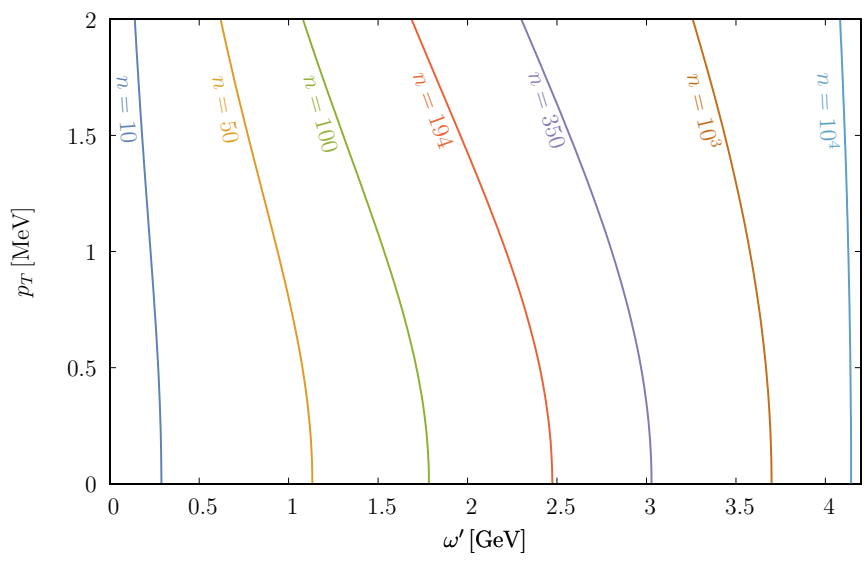

FIG. 6. Shift of the emission frequencies $\omega_{n}^{\prime}$ along the negative $z$ direction for different values of $n$ as a function of $p_{T}$ (vertical axis). The numerical parameters are $p_{z}=-4.2 \mathrm{GeV}$ and $I \approx 1.1 \times$ $10^{20} \mathrm{~W} / \mathrm{cm}^{2}$.

showing the explicit dependence also on $p_{T}^{2}$ (remember that now the energy $\varepsilon$ also depends on $p_{T}^{2}$ ). By expanding $\omega_{n}^{\prime}$ around $p_{T}=0$ we obtain

$$
\frac{\Delta \omega_{n}^{\prime}}{\omega_{n}^{\prime}}=\frac{1-(\varepsilon / n \omega-1) \zeta_{n}}{1+2 \zeta_{n}} \frac{\Delta p_{T}^{2}}{\varepsilon\left(\varepsilon-p_{z}\right)},
$$

where all the energies are calculated at $p_{T}=0$. This equation shows that again the relative shift depends on the harmonic number $n$. In a typical scenario where $\varepsilon \approx\left|p_{z}\right|$ and $\xi \gg 1$, the same approximations as in the previous section can be applied. The result for $\Delta \omega_{n}^{\prime}$ reads

$$
\Delta \omega_{n}^{\prime}=\zeta_{n} \frac{1+\zeta_{n}-\varepsilon \chi /\left(\xi^{3} \omega\right)}{\left(1+2 \zeta_{n}\right)^{2}} \frac{\Delta p_{T}^{2}}{\varepsilon},
$$

with $\zeta_{n}$ given in Eq. (24), which in the current approximations $\left(\varepsilon \approx\left|p_{z}\right|, \xi \gg 1\right)$ is approximately equal to $n \chi / \xi^{3}$. Equation (30) shows that an important role is played by the parameter $\mu=\varepsilon \chi /\left(\xi^{3} \omega\right)$. If we work in the quantum regime where $\chi \sim 1$, since at $\xi \sim 10^{2}$ electron energies in the $\mathrm{GeV}$ range are required, we can safely assume that $\mu \gg 1$. Moreover, at $\zeta_{n} \gg 1$ the emission spectrum is suppressed [4] such that we can conveniently further approximate the expression for $\Delta \omega_{n}^{\prime}$ as

$$
\Delta \omega_{n}^{\prime}=-\frac{\varepsilon \chi}{\xi^{3} \omega} \frac{\zeta_{n}}{\left(1+2 \zeta_{n}\right)^{2}} \frac{\Delta p_{T}^{2}}{\varepsilon} .
$$

This expression indicates that we would expect a negative shift of the harmonics, which becomes less pronounced at $\zeta_{n} \ll$ 1 (low harmonics) and at $\zeta_{n} \gg 1$ (high harmonics). This is exactly what we observe in Fig. 6, where different curves $\omega_{n}^{\prime}=$ $\omega_{n}^{\prime}\left(p_{T}\right)$ for different values of $n$ are plotted, with the numerical parameters: $p_{z}=-4.2 \mathrm{GeV}$ and $I \approx 1.1 \times 10^{20} \mathrm{~W} / \mathrm{cm}^{2}(\xi=$ $5, \chi \approx 0.25$, and $\mu \approx 5.4 \times 10^{6}$ ).

A typical collection of monochromatic electron spectra along the forward direction is shown in Fig. 7 by electrons having initially $p_{z}=-4.2 \mathrm{GeV}$ and either $p_{y}=0$ or $p_{x}=0$ [we recall that $p_{x}\left(p_{y}\right)$ is the component of the momentum along the direction of the electric (magnetic) field of the 


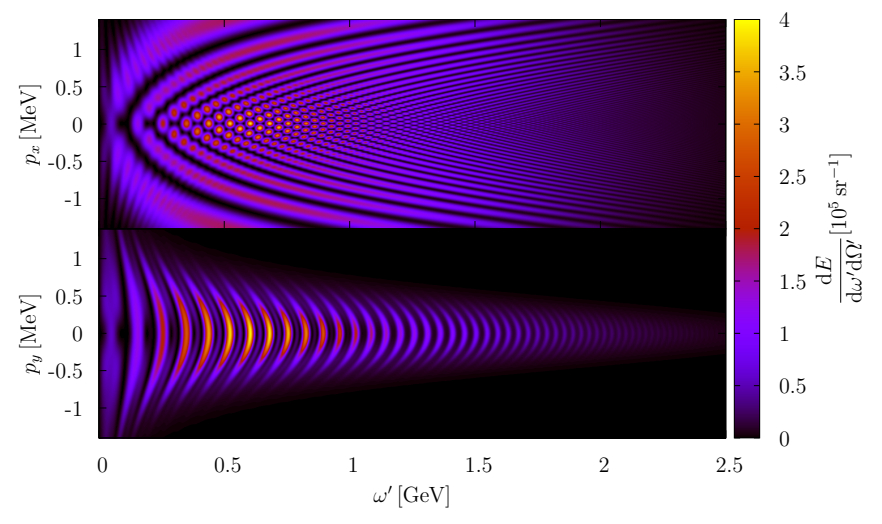

FIG. 7. Emission spectra in the negative $z$ direction for electrons having initially $p_{z}=-4.2 \mathrm{GeV}$ and either $p_{y}=0$ or $p_{x}=0$, after the interaction with a short laser pulse with $I \approx 1.1 \times 10^{20} \mathrm{~W} / \mathrm{cm}^{2}$.

laser], interacting with a short laser pulse with $I \approx 1.1 \times$ $10^{20} \mathrm{~W} / \mathrm{cm}^{2}(\xi=5, \chi \approx 0.25)$.

Apart from exhibiting the already-mentioned shift of the peak frequencies as one of the transverse components varies, we also observe that, by varying $p_{y}$ by about one to two electron masses, the spectrum is significantly suppressed. The reason is that the observation direction is the forward direction and that the angular emission range of the electron along the magnetic field of the laser is of about $m / \varepsilon$, whereas along the electric field of the laser, the electron emits up to angles of the order of $m \xi / \varepsilon$ [48]. It is also worth observing the large oscillations in the emitted intensity between successive peaks when varying $p_{x}$ (top part of Fig. 7). These oscillations are expected to have an important effect, when averaging many spectra, even for $\left|p_{x}\right| \ll m \xi$.

The above observations are confirmed by numerical calculations. In Fig. 8 (Fig. 9), we show the effects on the spectrum of the emitted photon along the negative $z$ direction [in a direction that lies on the $x z$ plane, the laser polarizationpropagation plane, and forms an angle $\theta=m \xi /(2 \bar{\varepsilon})$ with the negative $z$ axis, where $\bar{\varepsilon}$ is the average initial electron energy] of having either $\sigma_{p_{T}} \neq 0$ or $\sigma_{p_{z}} \neq 0$, or $\sigma_{p_{T}}, \sigma_{p_{z}} \neq 0$ [in the

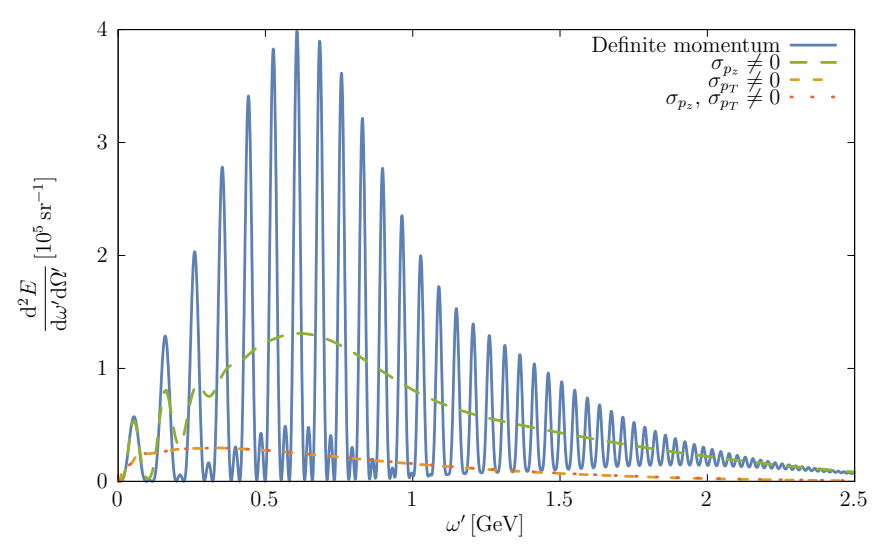

FIG. 8. Energy emission spectrum in the negative $z$ direction, for some different initial electron states. Here, $\overline{\boldsymbol{p}}=(0,0,-4.2 \mathrm{GeV})$, $\sigma_{p_{T}}=3 \times 10^{-4}|\overline{\boldsymbol{p}}|$, and $\sigma_{p_{z}}=6 \times 10^{-2}|\overline{\boldsymbol{p}}|$. The intensity of the laser field is $I \approx 1.1 \times 10^{20} \mathrm{~W} / \mathrm{cm}^{2}$.

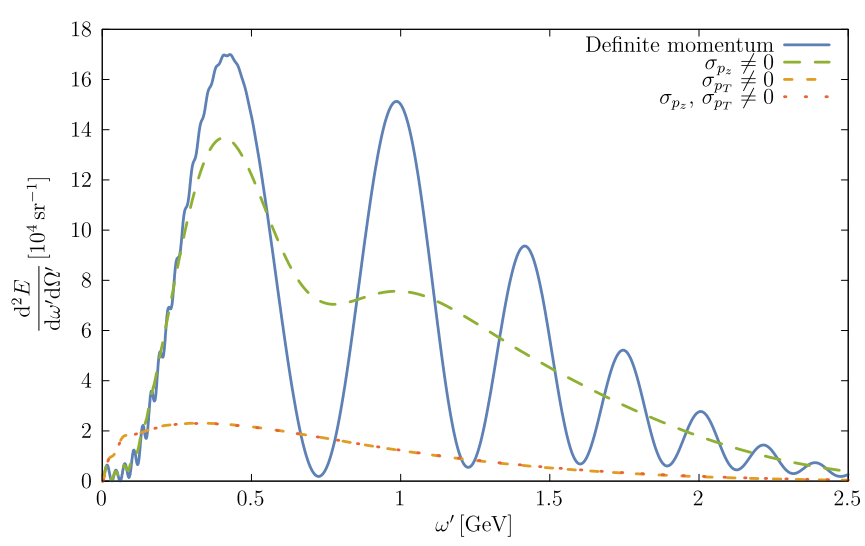

FIG. 9. Energy emission spectrum on a direction in the $x z$ plane forming an angle $\theta=m \xi / 2 \bar{\varepsilon}$ with the negative $z$ axis, for some different initial electron states. The numerical parameters are the same as in Fig. 8.

first two cases $\sigma_{p_{z}}$ and $\sigma_{p_{T}}$, respectively, are considered to be sufficiently small that their effects can be neglected as explained below Eq. (22)].

In the numerical spectra in Figs. 8 and 9, the average initial momentum of the electron is $\overline{\boldsymbol{p}}=(0,0,-4.2 \mathrm{GeV})$, and the indeterminacy on the transverse components is $\sigma_{p_{T}}=3 \times 10^{-4}|\overline{\boldsymbol{p}}|$, while the one on the $z$ component is $\sigma_{p_{z}}=6 \times 10^{-2}|\overline{\boldsymbol{p}}|$ (these parameters for the electron beam are compatible with those in Ref. [42]). The intensity of the laser field is $I \approx 1.1 \times 10^{20} \mathrm{~W} / \mathrm{cm}^{2}(\xi=5, \chi=\bar{\chi} \approx 0.25$ as calculated from the average electron momentum).

In Figs. 8 and 9 one can see that, for the chosen values of the parameters $\sigma_{p_{T}}$ and $\sigma_{p_{z}}$, the most dramatic alteration of the spectrum is due to the transverse momentum spread of the electron beam, even though its value is orders of magnitude smaller than the spread on $p_{z}$. In fact, the effect due to $\sigma_{p_{T}} \neq 0$ is so dominant that also switching on the longitudinal spread $\sigma_{p_{z}}$ has no observable effect on the emitted spectrum (the dotted red curve is on top of the short-dashed orange curve in both Figs. 8 and 9). As a result, the finer structures in the spectra are washed out and, in this respect, in order to at least partially observe them one should experimentally render the incoming electron beam as collimated as possible.

We also show in Fig. 10 the energy emission along a direction that lies on the laser polarization plane and forms an angle $m \xi /(2 \bar{\varepsilon})$ with the negative $z$ axis for $\chi=\bar{\chi} \approx 0.85$ (the parameters used for Fig. 10 are the same as for Fig. 9, except that $I \approx 1.2 \times 10^{21} \mathrm{~W} / \mathrm{cm}^{2}$ corresponding to $\xi=17$ ); the qualitative behavior for nonzero values of $\sigma_{p_{z}}$ and $\sigma_{p_{T}}$ is the the same as that previously discussed. We should emphasize that, as already mentioned in the discussion below Eq. (31), the larger effect due to the transverse momentum uncertainty is also related to the fact that the considered spectra refer to some specific observation directions. In fact, if we integrate with respect to the emission angles the spectrum corresponding to the numerical parameters in Fig. 10, we obtain the results in Fig. 11; they show that the total emitted energy as a function of $\omega^{\prime}$ changes only at frequencies $\omega^{\prime} \approx \bar{\varepsilon}=4.2 \mathrm{GeV}$ and that it is almost not affected by the momentum spreading of the incoming wave packet. The higher rates observed 


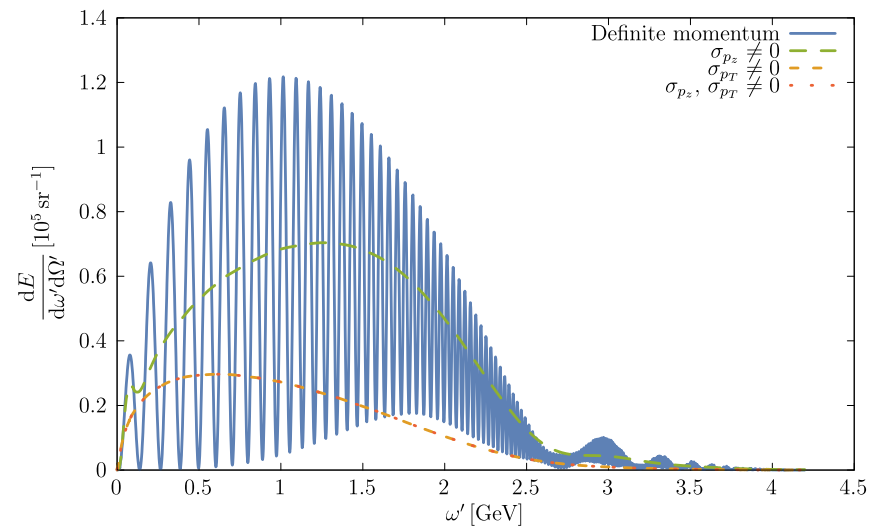

FIG. 10. Energy emission spectrum for an electron wave packet in the quantum regime $(\chi \approx 0.85)$, in the direction that lies on the laser polarization plane and forms an angle $m \xi /(2 \bar{\varepsilon})$ with the negative $z$ axis. The numerical parameters are the same as in Fig. 8, except that $I \approx 1.2 \times 10^{21} \mathrm{~W} / \mathrm{cm}^{2}$.

at these frequencies in the case of a wave packet with $\sigma_{p_{z}} \neq 0$ (see inset of Fig. 11) can be explained as some components of the wave packet have energies larger than $\bar{\varepsilon}$.

In order to analyze the properties of the emitted radiation in the spatial domain, one can integrate $d E / d \omega^{\prime} d \Omega^{\prime}$ with respect to $\omega^{\prime}$ and obtain the total energy emitted along each direction. A typical result of this procedure is shown in Fig. 12. On the lower panel the energy emitted per steradian by an electron in a Gaussian wave packet is plotted (the numerical parameters are the same as in Fig. 10). The upper panel shows the same quantity but emitted by an electron in a Volkov state with a definite momentum given by the $\overline{\boldsymbol{p}}$ of the mentioned Gaussian wave packet. In Fig. 12 the polar angle $\theta$ and the azimuthal angle $\phi$ are indicated assuming the negative $z$ axis as the polar axis.

As mentioned above, when the electron is initially in a pure Volkov state, and the laser is linearly polarized, the angular aperture of the emitted radiation is $m \xi / \varepsilon(m / \varepsilon)$ along the polarization (magnetic-field) direction, which is confirmed

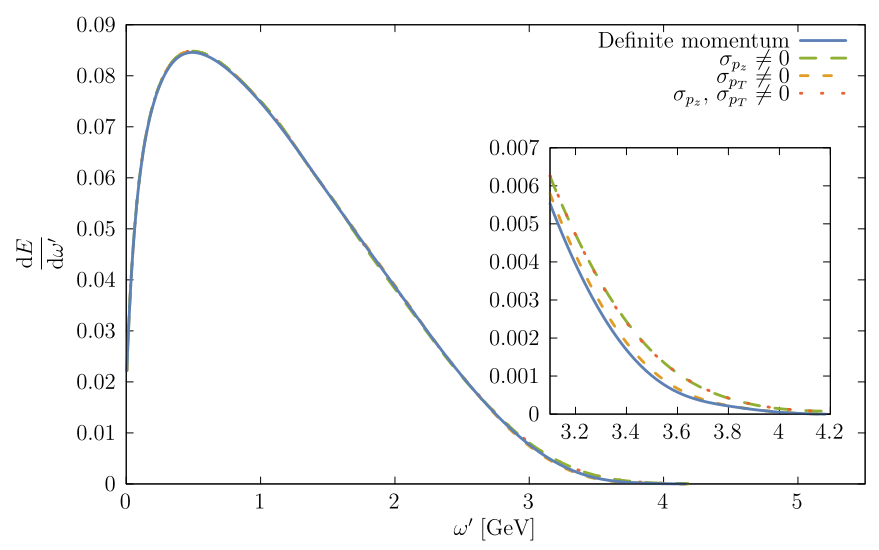

FIG. 11. Distribution of the total emitted energy by an electron in a Volkov state or in a Gaussian wave packet as a function of the frequency of the emitted photon. All the numerical parameters for this figure are the same as in Fig. 10.
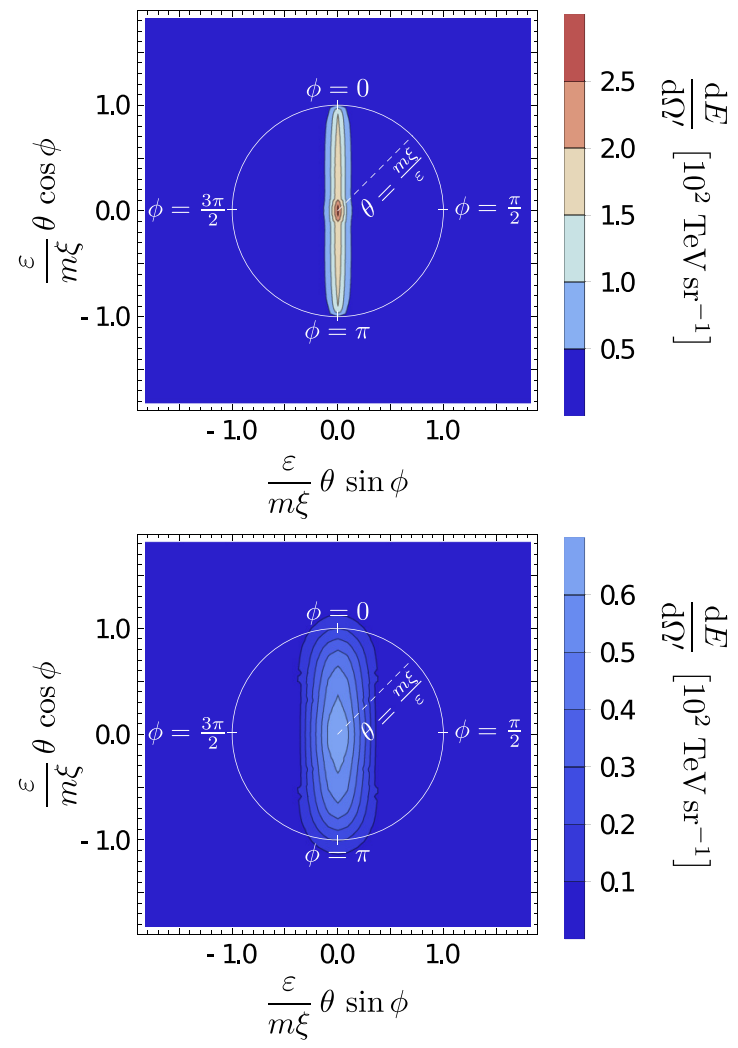

FIG. 12. Angular distribution of the total energy emitted by an electron in a Volkov state (upper panel) or in a Gaussian superposition of them (lower panel) after interacting with a strong laser field. The numerical parameters used here are the same as in Fig. 10.

by the upper panel in Fig. 12. The emission in the case of a multivariate Gaussian wave packet, in the lower panel of Fig. 12, extends over a broader region and is thus less intense, in the regime where $\sigma_{p_{T}}$ and $\sigma_{p_{z}}$ are much smaller than $\left|\bar{p}_{z}\right| \gg m$. In fact, at $\xi \gg 1$, if $\sigma_{p_{T}} \ll\left|\bar{p}_{z}\right|$ and $\sigma_{p_{z}} \ll\left|\bar{p}_{z}\right|$, the total energy emitted when the electron is either in a Volkov state or in a Gaussian wave packet is almost the same (see Fig. 11). Then, as the region of emission becomes broader, the radiation intensity in the Gaussian-wave-packet case decreases. We briefly notice here that this effect might also be exploited in principle as a diagnostic tool of the momentum spreading of the electron beam, provided that the laser parameters, such as its intensity, are known with sufficiently high accuracy.

\section{CONCLUSIONS}

In the present article we studied nonlinear single Compton scattering by an incoming electron described by a wave packet of Volkov states. We obtained that conservation of energy and momentum forbids interference effects among different momentum components of the wave packet, even if the electron is originally in a superposition of Volkov states. This means that an incoming electron wave packet can be equivalently described in this respect as a superposition of states or as a statistical mixture. The net effect of having a wave packet as initial electron state is a lowering and a smoothing of the angular-resolved emission spectrum for an electron in a state with definite momentum; this effect tends to be 
more pronounced than the nonmonochromaticity of the laser pulse (at comparable relative uncertainties in the electron and in the laser-photon energy). Furthermore, for realistic values of the properties of the electron wave packet as compared with those available experimentally for electron beams, the transverse momentum spread, even if orders of magnitude smaller than the longitudinal momentum spread, dominates the alterations on the structures and on the shape of the emission spectrum at a fixed observation direction. We observed that a broadening of the angular emission region also takes place in the case of an electron wave packet with respect to the case of a monoenergetic electron. However, by integrating the spectra over the observation directions, their dependence on the spreading of the initial wave packet is strongly suppressed.
[1] J. D. Jackson, Classical Electrodynamics (Wiley, New York, 1999).

[2] M. E. Peskin and D. V. Schroeder, Introduction to Quantum Field Theory (Addison-Wesley, Reading, 1995).

[3] V. B. Berestetskii, E. M. Lifshitz, and L. P. Pitaevskij, Quantum Electrodynamics (Elsevier Butterworth-Heinemann, Oxford, 1982).

[4] V. I. Ritus, J. Russ. Laser Res. 6, 497 (1985).

[5] A. Di Piazza, C. Müller, K. Z. Hatsagortsyan, and C. H. Keitel, Rev. Mod. Phys. 84, 1177 (2012).

[6] F. Ehlotzky, K. Krajewska, and J. Z. Kamiński, Rep. Prog. Phys. 72, 046401 (2009).

[7] F. Sauter, Eur. Phys. J. A 69, 742 (1931).

[8] H. W. Heisenberg and H. Euler, Eur. Phys. J. A 98, 714 (1936).

[9] J. Schwinger, Phys. Rev. 82, 664 (1951).

[10] L. S. Brown and T. W. B. Kibble, Phys. Rev. 133, A705 (1964).

[11] I. I. Goldman, Sov. Phys. JETP 46, 1412 (1964).

[12] A. I. Nikishov and V. I. Ritus, Sov. Phys. JETP 19, 529 (1964).

[13] D. Y. Ivanov, G. L. Kotkin, and V. G. Serbo, Eur. Phys. J. C 36, 127 (2004).

[14] M. Boca and V. Florescu, Phys. Rev. A 80, 053403 (2009).

[15] C. Harvey, T. Heinzl, and A. Ilderton, Phys. Rev. A 79, 063407 (2009).

[16] F. Mackenroth, A. Di Piazza, and C. H. Keitel, Phys. Rev. Lett. 105, 063903 (2010).

[17] M. Boca and V. Florescu, Eur. Phys. J. D 61, 449 (2011).

[18] F. Mackenroth and A. Di Piazza, Phys. Rev. A 83, 032106 (2011).

[19] D. Seipt and B. Kämpfer, Phys. Rev. A 83, 022101 (2011).

[20] K. Krajewska and J. Z. Kamiński, Phys. Rev. A 85, 062102 (2012).

[21] M. Boca, V. Dinu, and V. Florescu, Phys. Rev. A 86, 013414 (2012).

[22] M. Boca, V. Dinu, and V. Florescu, Nucl. Instrum. Methods Phys. Res., Sect. B 279, 12 (2012).

[23] T. N. Wistisen, Phys. Rev. D 90, 125008 (2014).

[24] D. Seipt, S. G. Rykovanov, A. Surzhykov, and S. Fritzsche, Phys. Rev. A 91, 033402 (2015).

[25] K. Krajewska, F. Cajiao Vélez, and J. Z. Kamiński, Phys. Rev. A 91, 062106 (2015).
[26] J. Peatross, C. Müller, K. Z. Hatsagortsyan, and C. H. Keitel, Phys. Rev. Lett. 100, 153601 (2008).

[27] C. Pellegrini, A. Marinelli, and S. Reiche, Rev. Mod. Phys. 88, 015006 (2016).

[28] W. H. Furry, Phys. Rev. 81, 115 (1951).

[29] J. P. Corson, J. Peatross, C. Müller, and K. Z. Hatsagortsyan, Phys. Rev. A 84, 053831 (2011).

[30] J. P. Corson and J. Peatross, Phys. Rev. A 84, 053832 (2011).

[31] D. Strickland and G. Mourou, Opt. Commun. 56, 219 (1985).

[32] A. Piskarskas, A. Stabinis, and A. Yankauskas, Sov. Phys. Usp. 29, 869 (1986).

[33] http://www.clf.rl.ac.uk/Facilities/Vulcan/12248.aspx.

[34] http://www.clf.stfc.ac.uk/CLF/Facilities/Astra/12254.aspx.

[35] http://cuos.engin.umich.edu/researchgroups/hfs/facilities/ hercules-petawatt-laser/.

[36] http://loasis.lbl.gov/.

[37] http://www.eli-laser.eu/.

[38] http://www.hiperlaser.org/.

[39] http://cilexsaclay.fr/Phocea/Vie_des_labos/Ast/ast_technique. php?id_ast=9.

[40] http://www.xcels.iapras.ru/.

[41] V. Malka, J. Faure, Y. A. Gauduel, E. Lefebvre, A. Rousse, and K. T. Phuoc, Nat. Phys. 4, 447 (2008).

[42] W. P. Leemans, A. J. Gonsalves, H.-S. Mao, K. Nakamura, C. Benedetti, C. B. Schroeder, Cs. Tóth, J. Daniels, D. E. Mittelberger, S. S. Bulanov, J.-L. Vay, C. G. R. Geddes, and E. Esarey, Phys. Rev. Lett. 113, 245002 (2014).

[43] L. D. Landau and E. M. Lifshitz, The Classical Theory of Fields (Elsevier, Oxford, 2013).

[44] A. Di Piazza, Phys. Rev. Lett. 113, 040402 (2014).

[45] L. N. G. Filon, Proc. R. Soc. Edinb. 49, 38 (1930).

[46] A. Iserles and S. P. Nørsett, Proc. R. Soc. London, Ser. A 461, 1383 (2005).

[47] D. Volkov, Eur. Phys. J. A 94, 250 (1935).

[48] F. Mackenroth, Quantum Radiation in Ultra-Intense Laser Pulses (Springer, Heidelberg, 2014).

[49] P. Baum, Chem. Phys. 423, 55 (2013). 